\title{
Zebrafish: An in vivo model for the study of human diseases
}

\author{
Bibhas Kar, Sivamani Subbiah \\ Center for Genetic Studies \& Research, the Madras Medical Mission, Chennai - 600037, India
}

Email address:

drbibhas_kar@yahoo.co.in (B. Kar)

\section{To cite this article:}

Bibhas Kar, Sivamani Subbiah. Zebrafish: An in Vivo Model for the Study of Human Diseases. International Journal of Genetics and Genomics. Vol. 1, No. 1, 2013, pp. 6-11. doi: 10.11648/j.ijgg.20130101.12

\begin{abstract}
The zebrafish (Danio rerio) is a powerful model organism for the study of vertebrate biology, being well suited to both developmental and genetic analysis. More recently, the rapid progress of various zebrafish genomics infrastructure initiatives is facilitating the development of zebrafish models of human disease. Genome organization and the pathways involved into control of signal transduction appear to be highly conserved between zebrafish and humans and therefore zebrafish may be used for modeling of human diseases. This review will highlight and describe the utility of zebrafish in the study of human diseases.
\end{abstract}

Keywords: Zebrafish, Genetics, Development, Human Diseases

\section{Introduction}

The zebrafish, a small tropical fish native to the rivers of India and South Asia [1], has become one of the most popular model organisms in developmental genetics and toxicology [2-6]. A number of unique features have contributed to its attraction, such as its generation time is short and most importantly a single spawning can produce hundreds of offspring [7]. In addition, maintenance costs are considerably lower than those for mammals. The entire body plan established by 24 hours post fertilization (hpf) and most of the internal organs like heart, liver, kidney and intestine totally developed by $96 \mathrm{hpf}$ [8]. Zebrafish larvae are transparent which means organs, cells and tissues are visualized in-vivo and investigated in real time $[9,10]$. The zebrafish is amenable to molecular and genetic analysis through rapid determination of temporal and spatial gene expression, examination of specific gene function by transgenic development, antisense gene knockdown and through large scale mutagenesis [11, 12]. Zebrafish have cardiovascular, nervous and digestive systems that are similar to those of mammals. Furthermore, a high degree of conservation exists between the human and zebrafish genomes (approximately 75\% similarity) [13-15]. Due to above advantages, zebrafish is the most important research tool today.

The last decade, zebrafish has been successfully applied as an organism to elucidate the etiology of human disease.
Zebrafish models of human disease are widely used in many different fields of medical research, like cancer, infectious diseases, cardiovascular disease, kidney disease, diabetes, blindness, deafness, digestive diseases, hematopoiesis, muscle disorders and neural disorders [16].

Below, selected examples illustrate the distinct uses of zebrafish technology to create a model, explore disease biology and to find new therapies.

\section{Disease Models}

\subsection{Cardiac Disease}

The embryonic heart of zebrafish closely resembles that of a human embryo at approximately three weeks gestation, being divided into atrial and ventricular chambers and lined by endocardium, with cardiac valves forming at the chamber boundaries [17]. Beating of the heart commences as a peristaltic wave at around $22 \mathrm{hpf}$, giving way to coordinated contractions of the atrium and ventricle by 36 hpf. Zebrafish are not dependent on circulation for survival at early stages of development. Therefore, perturbations in heart development do not result in the immediate death of the embryo. Coupled with the ease of visualization of the heart, this makes the zebrafish an especially attractive cardiac model. 
A range of zebrafish mutants have been identified with defects in cardiac development and/or function [17, 18]. In the mutant Pandora, the heart valve is absent; while in jekyll the entire ventricle is absent. In heart and soul, which has a lesion in a PKC lambda gene [19], heart size is greatly reduced, while in santa it is four times larger than normal. In miles apart there is no fusion of the two primitive heart tubes, leading to the formation of two cardiac structures on either side of the midline. In contrast, the mutant's passive aggressive, pickwick and hal affect contractility, while slow mo, trembler and reggae exhibit defects in rhythmicity. The slow mo mutant has a bradycardia, with a heart rate slower than wild type fish [20], with the mutation decreasing pacemaker current [21]. Another mutant, gridlock, resembles the human congenital disorder coarctation of the aorta [22] there is a lack of blood circulation to the tail, caused by a localized vascular defect in which the paired lateral dorsal aorta fail to fuse and instead generate a single dorsal aorta in the anterior trunk. This leads to the development of a collateral circulation, which permits survival [23]. Recently, Zhong et al. have shown that the gene responsible for gridlock encodes a basic helix-loop-helix protein of the Hairy/Enhancer of split related family genes [24], a family whose function is unknown. These examples again highlight the usefulness of zebrafish for gene discovery. A more recent mutagenesis screen has identified greater than 20 mutants with perturbations in cardiac induction and/or patterning using specific cardiac gene markers [25]. The identification of the underlying genetic lesions responsible for these mutants will likely provide further molecular details of these important developmental processes.

\subsection{Alzheimer's Disease}

Alzheimer's disease (AD) is the most common cause of dementia, with nearly $50 \%$ of dementia cases worldwide being attributed to $\mathrm{AD}$. The prevalence increases from $1.53 \%$ of the population between $65-69$ years to as high as $30 \%$ between $80-85$ years $[26,27]$. AD is characterized histopathologically by amyloid-beta $(\mathrm{A} \beta)$ containing plaques and intracellular neurofibrillary tangles consisting of abnormally phosphorylated tau protein in the brain [28, 29]. Cognitive impairments manifest themselves as progressive episodic memory loss and effects on executive functions, which are usually accompanied by mini-mental state examination scores below 24 [30]. Zebrafish possess two homologues of amyloid precursor protein (APP) with good (about 70\%) homology to human APP [31]. Studies have demonstrated the presence of the functional $\gamma$-secretase machinery to produce $\mathrm{A} \beta$ in zebrafish [32]. Other studies have investigated the tau protein in zebrafish [33]. Microinjection of four repeats human tau GFP constructs into 1-2 cell stage embryos showed disruption to the cytoskeletal structure and tau trafficking by 48 hours post injection. This eventually led to hyperphosphorylated fibrillar tau staining similar to that seen with neurofibrillary tangles in pathology of $\mathrm{AD}$ patients. These models offer the ability to screen for novel therapeutics that decrease $A \beta$ load and decrease the hyperphosphorylation seen in tauopathies.

\subsection{Muscular Dystrophy}

Duchenne muscular dystrophy (DMD) affects 1 in 3500 males and causes progressive muscle degeneration that can lead to death. DMD is the result of mutations in the sarcolemmal protein dystrophin located on the X-chromosome [50]. During an N-Ethyl-N-Nitrosourea (ENU) screen of zebrafish [94], a mutation referred to as sapje [51] was discovered. This mutation was later found to be located in the zebrafish DMD gene and causes progressive muscular degeneration in zebrafish larvae [52].

A model for muscular dystrophy was designed by using morpholinos to inactivate the gene encoding dystroglycan, a component of the dystrophin glycoprotein complex [34]. While mouse dystroglycan mutations result in early embryonic lethality, loss of dystroglycan function in the zebrafish is less severe. Embryos survive for at least 2 days, exhibiting muscle degeneration that resembles human muscular dystrophy. Genes coding for proteins linked to the familial DMD, dystrophin and Dp71 have also been cloned in zebrafish [35].

\subsection{Kidney Disorder}

Zebrafish have become a popular model for studying renal diseases thanks to the anatomical simplicity of their kidneys. Polycystic kidney disease (PKD), nephronophthisis, acute kidney injury (AKI), and a range of ciliopathies have been modeled in zebrafish [36]. Several studies suggest that cilia (microtubule based hair like organelles) play a central role in the etiology of PKD. The proper function of cilia prevents cystic formation and this hypothesis has been largely supported by the characterization of several zebrafish mutants carrying mutations in cilia proteins, such as intraflagellar transport proteins and LRRC50 [37-39]. Several ciliopathies including Bardet Biedl syndrome (BBS), nephronophthisis (NPHP), Jeune, Joubert, oro-facial-digital (OFD1) and Meckel syndromes (MKS) have been modeled in zebrafish using morpholinos for ciliopathy candidate genes. The use of drugs such as rapamycin and roscovitine ameliorate the renal phenotype observed in these morphant embryos, suggesting that zebrafish can be used to identify potential therapeutic agents for renal cystitis [40]. The zebrafish kidney is also a valuable system for studying AKI because, as opposed to mammals, fish can generate new nephrons throughout their life and regenerate new nephrons after injury [41]. Diep et al. in a series of transplantation experiments were able to identify LIM homeobox 1a-positive (lhxla-positive) cells as adult self renewing nephron stem/progenitor cells [41]. These findings pave the way for isolating similar cells in mammals with the aim of developing novel renal regenerative therapies. 


\subsection{Diabetes Mellitus}

Study of glucose metabolism is a good illustration of the metabolic models in zebrafish. Regulation of zebrafish glucose metabolism by insulin shares similarity with mammalian glucose metabolism. The main protein components of the zebrafish insulin signaling system exhibit structure functional similarity to those in mammals [44]. It was shown that sensitivity of adult zebrafish to the effects of antidiabetic drugs is similar to that of mammals. Zebrafish shares high similarity with mammals in regulation of expression of phosphoenolpyruvate carboxykinase (the enzyme catalyzing one of the key stages of gluconeogenesis) by glucagon and insulin. Researchers in this field suggest using changes in expression of this enzyme as the marker for studies of the effects of antidiabetic drugs [45].

\subsection{Leukemia}

The mouse model system has brought about a great deal of insight in this field, it has certain limitations such as difficulty in mapping second site mutations which are necessary for leukemic malignancy and in performing forward genetic screens in a reasonable time scale and at a reasonable cost. To avoid such limitations, scientists have more recently been adapting the zebrafish model system to study leukemic progression.

The first of the leukemia models using zebrafish was a $\mathrm{T}$ cell leukemia induced by expressing a mouse c-Myc oncogene. To create zebrafish transgenic lines prone to leukemia, Langenau et al. [46] created a transgenic zebrafish combining a Rag2 enhancer/promoter element to drive c-Myc expression in $\mathrm{T}$ and $\mathrm{B}$ cells. c-Myc is a very potent oncogene which disrupts the normal cell cycle causing cell over-proliferation. Aberrant expression of $\mathrm{c}-\mathrm{Myc}$ is found in many human $\mathrm{T}$ and $\mathrm{B}$ cell leukemias and lymphomas. The rag2-c-Myc transgenic zebrafish had a tumor latency of approximately $50 \mathrm{dpf}$. These transgenic lines were found to have their kidney and spleen full of lymphoblasts, and there was leukemic infiltration of the gut, gills and fins. These tumors were shown to be transplantable, demonstrating their clonality and tumorgenicity, similar to that of human tumors. Unfortunately, due to rapid onset of leukemia in these transgenic zebrafish, Langenau et al. [46] were not able to perform modifier or small molecule screens for suppressors of the leukemic phenotype. Subsequently, an acute lymphoblastic leukemia (ALL) zebrafish model was developed using the human TEL-AML1 translocation fusion protein [47]. TEL-AML1 is formed by $t(12,21)$ chromosomal arrangement; as a consequence, precursor cells are unable to differentiate into pre-B cells in patients with this translocation. Approximately $3 \%$ of the transgenic zebrafish expressing TEL-AML1 ubiquitously developed ALL after an 8 to 12 month latency period. However, transgenic zebrafish lines expressing TEL-AML1 under the
Rag2 promoter never developed leukemia. Transgenic zebrafish that developed ALL had lymphoblastic infiltration of the brain, ovary, liver, muscle and a kidney full of blast cells. Leukemic cells that were transplanted into a wild type fish caused a similar phenotype to that of the donor zebrafish approximately 8 weeks post transplantation. The ALL observed in the transgenic zebrafish was reminiscent of ALL caused by the TEL-AML1 translocation in humans. This study was able to determine a potential pathway for TEL-AML1 Leukemogenesis as well as to identify some of the TEL-AML1 cellular targets [47]. Such knowledge leads to better understanding of the leukemic process and better treatments for patients with such translocation.

Very recently, an acute myeloid leukemia (AML) zebrafish model was created by expressing a human AML1-ETo translocation fusion gene. [48]. This model is similar to the type of zebrafish AML model that we have been trying to develop as well. This AML zebrafish model was developed by expressing human AML1-ETO fusion transgene using a heat shock promoter. Transgenic embryos were heat shocked causing expression of AML1-ETO, which resulted in accumulation of immature blast cells, lack of circulating blood cells, disruption of definitive hematopoiesis, and conversion of erythropoiesis to myelopoieis in the ICM. The phenotypes observed due to AML1-ETO expression in zebrafish embryos are similar in nature of the phenotype observed in patients with AML [49]. Thus far this model has lead to better understanding of AML1-ETO function in leukemic progression [48].

Although this zebrafish AML model is a great beginning in understanding AML leukemogenesis, further studies need to be done to confirm that transgenic zebrafish will also develop AML. It is necessary to confirm the embryonic phenotype in adult zebrafish because there are differences in pathways, cell types, and precursor cells, between primitive hematopoiesis and definitive hematopoiesis. A potential drawback of this model is that AML1-ETO is being expressed using a heat shock promoter, which allows for temporal control but results in ubiquitous expression of AML1-ETO. This might be a concern since it is possible that some of the embryonic phenotypes observed might be due in part to other tissues being affected besides the blood. It is also unforeseen which cells might to other tissues being affected besides the blood. It is also unforeseen which cells might be affected when AML1-ETO is expressed ubiquitous in adult zebrafish and if short periods on AML1-ETO expression would be sufficient to cause a leukemic phenotype in adult zebrafish.

\subsection{Alcoholic Fatty Liver Disease}

Alcoholic fatty liver disease (ALD) is estimated to be involved in over $50 \%$ of all deaths due to liver failure secondary to liver cirrhosis, but accurate estimates for prevalence are unavailable [53]. Binge drinking leads to transient fatty liver disease, but chronic alcohol 
consumption can lead to fibrosis, cirrhosis, and steatohepatitis [54]. An ALD model has also been developed in zebrafish with administration of $2 \%$ ethanol to the water of $4 \mathrm{dpf}$ zebrafish for 32 days. This regimen results in hepatomegaly and steatosis, alongside up regulation of hepatic cyp $2 \mathrm{e} 1$, sod (superoxide dismutase), and bip gene expression indicating hepatotoxic metabolism of the ethanol [55]. Importantly, ethanol induced steatosis was prevented by morpholino blockade of the sterol regulatory binding protein (Srebps). Because Srebps activation is important in chronic alcoholic liver disease [55] in humans, the zebrafish ALD model could be utilized to identify new therapeutic mechanisms and to screen for therapeutic agents.

\subsection{Non - Alcoholic Fatty Liver Disease}

The most characterized zebrafish model of Non-alcoholic fatty liver disease (NAFLD) involved a forward genetic screen using viral insertion and screening for hepatomegaly. In the case of the NAFLD model, a 172 bp gene trap cassette was found inserted in the intron between exons 11 and 12 of the recently discovered zebrafish gene foie gras (foigr) [56], which results in a frame shift mutation, and generation of a stop codon. This mutation (foigrhi1532b) results in the development of steatosis (fatty liver disease) resembling human NAFLD, characterized by large lipid filled hepatocytes and cellular apoptosis in larvae as young as $5 \mathrm{dpf}$ [56]. However, the exact function of this gene, which is highly conserved across species including humans, is not yet determined. Further studies with the foigr mutant have shown that the apoptosis observed involves increased ER stress and is regulated in part through the UPR gene atf6. Morpholino blockade of atf6 ameliorates liver injury during chronic ER stress in the foigr mutants [57]. However, atf6 blockade potentiates steatosis during acute ER stress induced by the toxin tunicamycin [57], suggesting that atf6 may have variable effects in different phases (acute/chronic) of liver injury. Hepatic steatosis has also been induced in zebrafish larvae using thioacetamide [58]. Investigators have shown that administration of $0.025 \%$ thioacetamide into the fish media at $3 \mathrm{dpf}$ resulted in liver damage by $5 \mathrm{dpf}$, characterized by increased accumulation of fatty droplets, hepatocyte apoptosis, and up regulation of apoptotic genes such as bad, bax, p-38a, caspase-3, 8 and jnk-1. Overall, the availability of NAFLD zebrafish models is burgeoning, and they are poised to be screened for pharmacological compounds that could for the first time effectively treat this disease.

\section{Conclusions}

The zebrafish is rapidly gaining acceptance as a promising animal model for human diseases which facilitate large cost effective studies that can be combined with battery of advanced molecular tools for genetic modulation and powerful in-vivo molecular visuals readouts to carry out forward and reverse genetics in a more efficient manner than possible with traditional models. In this review we have described the application of the zebrafish models to various human diseases. In summary, the zebrafish as a well established model organism, making important contributions to the identification and characterization of genes and pathways involved in development, organ function and behaviour. Additionally it has become a valuable resource for identifying genes involved in human disease.

\section{References}

[1] RC. Eaton, RD. Farley, "Spawning cycle and egg production of zebrafish (Brachydanio rerio) in the laboratory" Copeia. Vol. 1, pp. 195-209, 1974.

[2] LE. Coverdale, D. Lean, CC. Martin, "Not just a fishing trip-Environmental genomics using zebrafish" Curr. Genomics. Vol. 5, pp. 395-407, 2004.

[3] P. Goldsmith, "Zebrafish as a pharmacological tool: the how, why and when" Curr. Opin. Pharmacol. Vol. 4, pp. 504-512, 2004.

[4] AJ. Hill, H. Teraoka, W. Heideman, RE. Peterson, "Zebrafish as a model vertebrate for investigating chemical toxicity" Toxicol. Sci. Vol. 86, pp. 6-19, 2005.

[5] R. Nagel, "DarT: The embryotest with the zebrafish Danio rerio-a general model in ecotoxicology and toxicology" ALTEX. Vol. 19, pp. 38-48, 2002.

[6] C. Parng, WL. Seng, C. Semino, P. McGrath, "Zebrafish: a preclinical model for drug screening" Assay Drug Dev. Technol. Vol. 1, pp. 41-48, 2002.

[7] WH. Detrich, M. Westerfield, LI. Zon, "The zebrafish: Biology-Methods in cell biology" Vols. 59-60. San Diego: Academic Press, 1999.

[8] M. Westerfield, "The zebrafish book. A guide for the laboratory use of zebrafish (Danio rerio)" 4th ed.; Univ. of Oregon Press, Eugene, 2000.

[9] JS. Eisen, "Zebrafish makes a big splash” Cell. Vol. 87(6), pp. 969-977, 1996.

[10] MC. Fishman, "Zebrafish genetics: The enigma of arrival" Proc. Natl. Acad. Sci. USA Vol. 96(19), pp. 10554-10556, 1999.

[11] W. Driever, L. Solnica-Krezel, AF. Schier, SC. Neuhauss, J. Malicki, DL. Stemple, DY. Stainier, F. Zwartkruis, S. Abdelilah, Z. Rangini, J. Belak, C. Boggs, "A genetic screen for mutations affecting embryogenesis in zebrafish" Development. Vol. 123(1), pp. 37-46, 1996.

[12] P. Haffter, M. Granato, M. Brand, MC. Mullins, M. Hammerschmidt, DA. Kane, J. Odenthal, FJ. Van Eeden, YJ. Jiang, CP. Heisenberg, RN. Kelsh, M. Furutani-Seiki, E. Vogelsang, D. Beuchle, U. Schach, C. Fabian, C. Nusslein-Volhard, "The identification of genes with unique and essential functions in the development of the zebrafish, Danio rerio” Development. Vol. 123(1), pp. 1-36, 1996. 
[13] J. Cerda, M. Conrad, J. Markl, M. Brand, H. Herrmann, "Zebrafish vimentin: Molecular characterisation, assembly properties and developmental expression" Eur. J. Cell Biol. Vol. 77, pp. 175-187, 1998.

[14] JH. Postlethwait, IG. Woods, P. Ngo-Hazelett, YL. Yan, PD. Kelly, F. Chu, H. Huang, A. Hill Force, WS. Talbot, "Zebrafish comparative genomics and the origins of vertebrate chromosomes" Genome Res. Vol. 10, pp. 1890-902, 2000.

[15] Zebrafish Genome Resources [online], http://www.ncbi.nlm. nih.gov/genome/guide/zebrafish/

[16] A. Amsterdam, N. Hopkins, "Mutagenesis strategies in zebrafish for identifying genes involved in development and disease" Trends Genet. Vol. 22(9), pp. 473-8, 2006.

[17] KS. Warren, MC. Fishman, "Physiological genomics: mutant screens in zebrafish" Am. J. Physiol. Vol. 275, pp. H1-7, 1998.

[18] MC. Fishman, DY. Stainier, RE. Breitbart, M. Westerfield, "Zebrafish: genetic and embryological methods in a transparent vertebrate embryo" Methods Cell Biol. Vol. 52, pp. 67-82, 1997.

[19] S. Horne-Badovinac, D. Lin, S. Waldron, M. Schwarz, G. Mbamalu, T. Pawson, Y. Jan, DY. Stainier, S. Abdelilah Seyfried, "Positional cloning of heart and soul reveals multiple roles for PKClambda in zebrafish organogenesis" Curr. Biol. Vol. 11, pp. 1492-1502, 2001.

[20] K. Baker, KS. Warren, G Yellen, MC. Fishman, "Defective pacemaker current (Ih) in a zebrafish mutant with a slow heart rate" Proc. Nat. Acad. Sci. USA Vol. 94, pp. 4554-4559, 1997.

[21] KS. Warren, K. Baker, MC. Fishman, "The slow mo mutation reduces pacemaker current and heart rate in adult zebrafish" Am. J. Physiol. Heart Circ. Physiol. Vol. 281, pp. H1711-1719, 2001.

[22] JA. Towbin, TC. McQuinn, "Gridlock: a model for coarctation of the aorta?" Nat. Med. Vol. 1, pp. 1141-1142. 1995.

[23] BM. Weinstein, DL. Stemple, W. Driever, MC. Fishman. "Gridlock, a localized heritable vascular patterning defects in the zebrafish" Nat. Med. Vol. 1, pp. 1143-1147, 1995.

[24] TP. Zhong, M. Rosenberg, MA. Mohideen, B. Weinstein, MC. Fishman, "Gridlock, an HLH gene required for assembly of the aorta in zebrafish" Science Vol. 287, pp. 1820-1824, 2000 .

[25] J. Alexander, DY. Stanier, D. Yelon, "Screening mosaic F1 females for mutations affecting zebrafish heart induction and patterning” Dev. Genet. Vol. 22, pp. 288-299, 1998.

[26] R. Vandenberghe, J. Tournoy, "Cognitive aging and Alzheimer's disease" Postgrad. Med. J. Vol. 81, pp. 343-52, 2005 .

[27] C. Mount, C. Downton, "Alzheimer disease: progress or profit?" Nat. Med. Vol. 12, 780-4, 2006.

[28] DJ. Selkoe, "Toward a comprehensive theory for Alzheimer's disease. Hypothesis: Alzheimer's disease is caused by the cerebral accumulation and cytotoxicity of amyloid beta-protein" Ann. NY Acad. Sci. Vol. 924, pp.
$17-25,2000$.

[29] A. Mudher, S. Lovestone, "Alzheimer's disease-do tauists and baptists finally shake hands?" Trends Neurosci. Vol. 25, pp. 22-6, 2002.

[30] R. Vandenberghe, J. Tournoy, "Cognitive aging and Alzheimer's disease” Postgrad. Med. J. Vol. 81, pp. 343-52, 2005.

[31] A. Musa, H. Lehrach, VA. Russo, "Distinct expression patterns of two zebrafish homologues of the human APP gene during embryonic development" Dev. Genes. Evol. Vol. 211, pp. 563-7, 2001.

[32] M. Newman, FI. Musgrave, M. Lardelli, "Alzheimer disease: amyloidogenesis, the presenilins and animal models" Biochim. Biophys. Acta. Vol. 1772, pp. 285-97, 2007.

[33] HG. Tomasiewicz, DB. Flaherty, JP. Soria, JG Wood, "Transgenic zebrafish model of neurodegeneration" J. Neurosci. Res. Vol. 70, pp. 734-45, 2002.

[34] MJ. Parsons, I. Campos, EM. Hirst, DL. Stemple, "Removal of dystroglycan causes severe muscular dystrophy in zebrafish embryos" Development Vol. 129, pp. 3505-3512, 2002.

[35] F. Bolanos-Jimenez, A. Bordais, M. Behra, U. Strahle, D. Mornet, J. Sahel, A. Rendon "Molecular cloning and characterization of dystrophin and Dp71, two products of the Duchenne Muscular Dystrophy gene, in zebrafish" Gene. Vol 274, pp. 217-226, 2001.

[36] LM. Swanhart, CC. Cosentino, CQ. Diep, AJ. Davidson, M. de Caestecker, NA. Hukriede, "Zebrafish kidney development: basic science to translational research" Birth Defects Res. C. Embryo Today. Vol. 93(2), pp. 141-156, 2011.

[37] Y. Cao, A. Park, Z. Sun, "Intraflagellar transport proteins are essential for cilia formation and for planar cell polarity" J. Am. Soc. Nephrol. Vol. 21(8), pp. 1326-1333, 2010.

[38] Z. Sun, A. Amsterdam, GJ. Pazour, DG. Cole, MS. Miller, N. Hopkins, "A genetic screen in zebrafish identifies cilia genes as a principal cause of cystic kidney" Development. Vol. 131(16), pp. 4085-4093, 2004.

[39] E. Van Rooijen, RH. Giles, EE. Voest, C. Van Rooijen, S. Schulte-Merker, FJ. Van Eeden, "LRRC50, a conserved ciliary protein implicated in polycystic kidney disease" J. Am. Soc. Nephrol. Vol. 19(6), pp. 1128-1138, 2008.

[40] JL. Tobin, PL. Beales, "Restoration of renal function in zebrafish models of ciliopathies" Pediatr. Nephrol. Vol. 23(11), pp. 2095-2099, 2008.

[41] CQ. Diep, D Ma, RC. Deo, TM. Holm, RW. Naylor, N. Arora, RA. Wingert, F. Bollig, G. Djordjevic, B. Lichman, H. Zhu, T. Ikenaga, F. Ono, C. Englert, CA. Cowan, NA. Hukriede, RI. Handin, AJ. Davidson, "Identification of adult nephron progenitors capable of kidney regeneration in zebrafish" Nature. Vol. 470(7332), pp. 95-100, 2011.

[42] IA. Drummond, A. Majumdar, H. Hentschel, M. Elger, L. Solnica-Krezel, AF. Schier, SC. Neuhauss, DL. Stemple, F. Zwartkruis, Z. Rangini, W. Driever, MC. Fishman, "Early development of the zebrafish pronephros and analysis of mutations affecting pronephric function" Development. Vol. 125, pp. 4655-4667, 1998. 
[43] GR. Dressler, "Kidney development branches out" Dev. Genet. Vol. 24, pp. 189-193, 1999.

[44] GS. Gerhard, "Comparative aspects of zebrafish (Danio rerio) as a model for aging research" Experimen. Gerontol. Vol. 38, pp. 1333-1341, 2003.

[45] B. Elo, CM. Villano, D. Govorco, LA. White, "Larval zebrafish as a model for glucose metabolism: expression of phosphoenolpyruvate carboxykinase as a marker for exposure to anti diabetic compounds" J. Mol. Endocinol. Vol. 38, pp. 433-440, 2007.

[46] M. David, Langenau, David Traver, A. Adolfo, Ferrando, L. Jeffery, Kutok, C. Jon, Aster, P. John, Kanki, Shuo Lin, Ed Prochownik, S. Nikolaus, Trede, I. Leonard, A. Zon, Thomas Look, "Myc-Induced T Cell Leukemia in Transgenic Zebrafish" Science. Vol. 299 (5608), pp. 887-890, 2003.

[47] E. Hatem, Sabaawy, Mizuki Azuma, J. Lisa, Embree, Huai-Jen Tsai, F. Matthew, Starost, Dennis D Hickstein, "TEL-AML1 transgenic zebrafish model of precursor B cell acute lymphoblastic leukemia" Proc. Natl. Acad. Sci. USA Vol. 103(41), pp. 15166-15171, 2006.

[48] JR. Yeh, KM. Munson, YL. Chao, QP. Peterson, CA. Macrae, RT. Peterson, "AML1-ETO reprograms hematopoietic cell fate by down regulating scl expression" Development. Vol. 135(2), pp. 401-410, 2008.

[49] KE. Elagib, AN. Goldfarb, "Oncogenic pathways of AML1-ETO in acute myeloid leukemia: multifaceted manipulation of marrow maturation" Cancer Lett. Vol. 251(2), pp. 179-86, 2007.

[50] AEH. Emery, "The muscular dystrophies" The Lancet. Vol. 359(9307), pp. 687-695, 2002.

[51] M. Granato, FJ. Van Eeden, U. Schach, T. Trowe, M. Brand,
M. Furutani-Seiki, P. Haffter, M. Hammerschmidt, CP. Heisenberg, YJ. Jiang, DA. Kane, RN. Kelsh, MC. Mullins, J. Odenthal, C. Nusslein-Volhard, "Genes controlling and mediating locomotion behavior of the zebrafish embryo and larva” Development. Vol. 123, pp. 399-413, 1996.

[52] DI. Bassett, RJ. Bryson-Richardson, DF. Daggett, P. Gautier, DG. Keenan, PD. Currie. "Dystrophin is required for the formation of stable muscle attachments in the zebrafish embryo" Development. Vol. 130(23), pp. 5851-5860, 2003.

[53] WR. Kim, RS. Brown, NA. Terrault, H. El-Serag, "Burden of liver disease in the United States: summary of a workshop" Hepatology, Vol. 36(1), pp. 227-242, 2002.

[54] CS. Lieber, "Alcoholic fatty liver: its pathogenesis and mechanism of progression to inflammation and fibrosis" Alcohol. Vol. 34(1), pp. 9-19, 2004.

[55] MJ. Passeri, A. Cinaroglu, C. Gao, KC. Sadler, "Hepatic steatosis in response to acute alcohol exposure in zebrafish requires sterol regulatory element binding protein activation" Hepatology, Vol. 49(2), pp. 443-452, 2009.

[56] KC. Sadler, A. Amsterdam, C. Soroka, J. Boyer, and N. Hopkins, "A genetic screen in zebrafish identifies the mutants vps18, nf2 and foie gras as models of liver disease" Development. Vol. 132(15), pp. 3561-3572, 2005.

[57] A. Cinaroglu, C. Gao, D. Imrie, KC. Sadler, "Activating transcription factor 6 plays protective and pathological roles in steatosis due to endoplasmic reticulum stress in zebrafish" Hepatology, Vol. 54(2), pp. 495-508, 2011.

[58] AA. Amali, RD. Rekha, CJ. Lin, WL. Wang, HY. Gong, GM. Her, JL. Wu, "Thioacetamide induced liver damage in zebrafish embryo as a disease model for steatohepatitis" J. Biomed. Sci, Vol. 13(2), pp. 225-232, 2006. 